\title{
Time-resolved tunneling in the quantum Hall regime
}

\author{
N. C. van der Vaart, ${ }^{*}$ L. P. Kouwenhoven, M. P. de Ruyter van Steveninck, Y. V. Nazarov, and C. J. P. M. Harmans \\ Department of Applied Physics, Delta University of Technology, P.O. Box 5046, 2600 GA Delft, The Netherlands \\ C. T. Foxon \\ Philips Research Laboratories, Redhill, Surrey RH15HA, United Kingdom \\ (Received 29 April 1996; revised manuscript 27 December 1996)
}

\begin{abstract}
We have studied the quantum Hall effect in the mesoscopic regime by measuring in detail the scattering events between the two spin-resolved levels of the lowest orbital Landau level. Our sample consists of a micrometer-sized quantum dot that is weakly coupled to large two-dimensional electron gases. In a high magnetic field electron transport through the dot is regulated by a mixture of Coulomb blockade effects and Landau-level quantization. Some Coulomb blockade oscillations show switching as a function of time between two discrete conductance values. We show that each switch is a time-resolved measurement of a single electron tunnel event between two Landau levels. Upon increasing the magnetic field, the time between two tunnel events reaches values as large as $100 \mathrm{~s}$. We explain our observations with a phenomenological model that describes the dot as a two-level fluctuator. Furthermore, we have studied Coulomb oscillations as a function of magnetic field. The period of these magneto-Coulomb oscillations depends strongly on the occupation of the Landau levels in the reservoirs. [S0163-1829(97)06115-8]
\end{abstract}

\section{INTRODUCTION}

The quantum Hall effect arises in millimeter-sized twodimensional electron gases (2DEG's). The important observations are that the Hall resistance takes on quantized values $h / n e^{2}$ ( $n$ is an integer) and simultaneously the longitudinal resistance vanishes. ${ }^{1}$ These observations are a result of a strong reduction of the scattering probabilities of electrons moving in a 2D plane in a high perpendicular magnetic field. The electron transport can be conveniently described in terms of edge channels. ${ }^{2}$ Büttiker ${ }^{3}$ has developed an edgechannel-transport formalism that can also describe scattering. Experimentally, it was shown that electrons can travel over distances as long as a millimeter without being scattered between different edge channels. ${ }^{4}$ These experiments were all discussed in terms of noninteracting electrons. Recently, it has become clear that the self-consistent arrangement of charge is sometimes necessary to include for understanding the properties of the electron states at the sample edge. ${ }^{5-10}$ The emerging picture is that near the sample edge the quantum Hall states separate in alternating strips of incompressible and compressible states. This picture is also valid when the quantum Hall states are confined in a quantum dot. ${ }^{6,7}$

In this paper, we study single-electron-scattering events between different compressible regions. We use the Coulomb blockade of tunneling ${ }^{11}$ to control and measure singleelectron scattering events between two Landau levels. We find that the time between two tunnel events can be tuned to macroscopic values. This allows for time-resolved measurements of single-electron tunnel events. ${ }^{12}$ We show that a quantum dot provides a completely controllable two-level fluctuator, where the magnetic field controls the energy difference and the transition rate between the two levels.

This paper is organized as follows: In Sec. II we discuss our sample. Section III describes time-resolved measurement of single-electron tunneling events and in Sec. IV we discuss
Coulomb oscillations in the quantum Hall regime. Section V is devoted to the magnetic-field dependence of the typical time between two tunnel events and the role of the reservoirs on transport through the dot. In Sec. VI we summarize our conclusions.

\section{DEVICE DESCRIPTION}

Figure 1(a) shows a schematic of the gate geometry of our quantum dot. The light parts are metallic gates fabricated on top of a GaAs/Al $\mathrm{Al}_{x} \mathrm{Ga}_{1-x} \mathrm{As}$ heterostructure with a $2 \mathrm{DEG}$ $100 \mathrm{~nm}$ below the surface. We denote gate $F$ as the finger gate, gates 1 and 2 as the quantum point contact (QPC) gates, and gate $C$ as the center gate. Applying a negative voltage to gates $F, C, 1$, and 2 depletes the electron gas underneath them, and forms a quantum dot in the 2DEG with a diameter of roughly $600 \mathrm{~nm}$. Electron transport occurs via the tunnel barriers induced by gates 1 and $F$, and by 2 and $F$, which couple the dot to the two large 2DEG reservoirs. The number of electrons in the dot can be varied with the voltage $V_{C}$ applied to the center gate $C$.

The measurements presented in this paper were performed on three different samples, whose properties are summarized in Table I. Throughout most of the paper we neglect the presence of zero-dimensional energy states in the dot. Their separation is comparable to temperature. The experiments are performed in a dilution refrigerator with a base temperature of $10 \mathrm{mK}$. The temperature of the electron gas, however, is typically around $100 \mathrm{mK}$. We used a dcmeasurement technique to detect the current through the quantum dot in response to a dc source-drain voltage $V$ across the two electron reservoirs.

\section{DETECTION OF SINGLE ELECTRON TUNNEL EVENTS BETWEEN LANDAU LEVELS}

McEuen et al. ${ }^{6,7}$ have pointed out that the phase separation of quantum Hall states in a quantum dot leads to the 
(a)

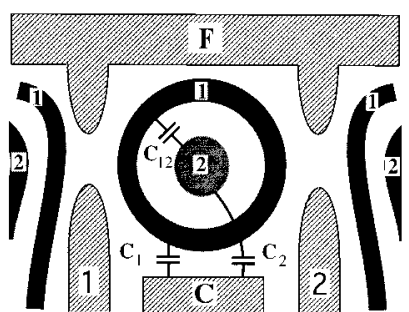

(b)

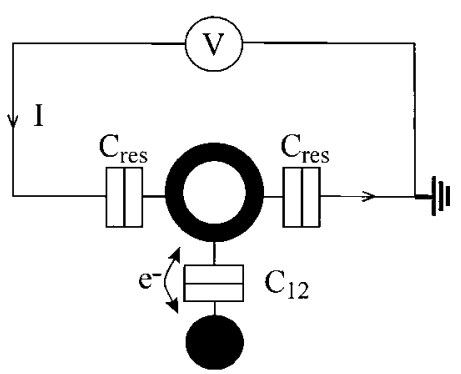

FIG. 1. (a) Schematic of the gate geometry. $F$ denotes the finger gate, 1 and 2 the quantum point contact gates, and $C$ the center gate. The locations of the compressible parts of $L L_{1}$ and $L L_{2}$ are denoted by the dark, hatched parts. $C_{1}\left(C_{2}\right)$ denotes the capacitance of $L L_{1}\left(L L_{2}\right)$ to its surroundings. $C_{12}$ is the inter-Landaulevel capacitance. See Ref. 12 for a photograph of the device. (b) Simplified electrical circuit of a dot with two Landau levels in the charging regime. A current $I$ flows through the ring in response to a dc voltage $V$. The ring can be used as an electrometer to measure the transfer of a single electron $e^{-}$between the core and the ring.

picture of Fig. 1(b). Self-consistent arrangement of the charge in a high magnetic field results in alternating regions of compressible and incompressible electron liquids. ${ }^{4-6}$ When only the two spin states of the lowest-orbital Landau level $\left(L L_{1}\right.$ and $\left.L L_{2}\right)$ are occupied, $L L_{2}$ forms a compressible core in the center of the dot and $L L_{1}$ is compressible only in the ring region around the core. The ring and the core are spatially separated by an incompressible quantum Hall fluid (i.e., the fully occupied $L L_{1}$ ), which acts as a tunnel barrier. This barrier separates the two partially filled Landau levels such that these can be treated as independent Coulomb islands. We can therefore divide the total number of electrons $N$ in the quantum dot in an integer $N_{1}$ electrons in $L L_{1}$ and an integer $N_{2}$ in $L L_{2}$ with $N=N_{1}+N_{2}$.

Transport from one 2DEG reservoir via the dot to the other reservoir primarily occurs via the ring, since the core is separated from the reservoirs over a much larger distance. ${ }^{13}$ Hence, we can represent a quantum dot with two confined

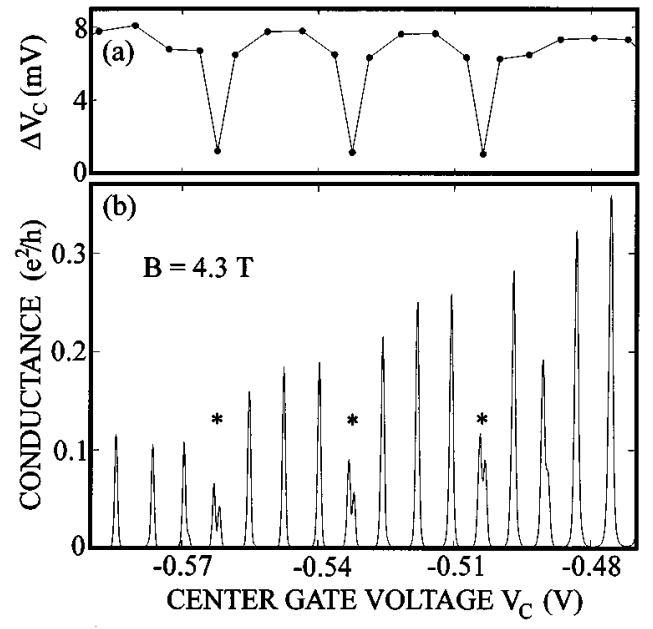

FIG. 2. (a) Peak spacing of the Coulomb oscillations in (b) versus gate voltage. (b) Coulomb oscillations as a function of the center gate voltage at a magnetic field of $4.3 \mathrm{~T}$. Measurements are performed on dot I.

Landau levels by the electrical circuit of Fig. 3, below. The ring is connected to the reservoirs via two tunnel barriers. The source-drain voltage $V$ drives a current $I$ through the ring in the dot. The core is connected to the ring via a tunnel barrier. Note that in an electrical circuit a tunnel barrier is usually represented as a resistor parallel to a capacitor.

Adding an electron to a small isolated region costs a finite charging energy. At zero magnetic field, only one charging energy is important. In a high magnetic field with $L L_{1}$ and $L L_{2}$ occupied, three charging energies are relevant. Adding an electron from the reservoirs to $L L_{1}$ increases the electrochemical potential $\mu_{1}$ of $L L_{1}$ by an amount $E_{1}$ and also increases the electrochemical potential $\mu_{2}$ by an interaction energy $E_{12}$. Similarly, adding an electron to $L L_{2}$ increases $\mu_{2}$ by $E_{2}$ and $\mu_{1}$ by $E_{12}$. Single-electron tunneling within the dot keeps the total number $N$ constant. However, removing an electron from $L L_{2}$ and putting it in $L L_{1}$ increases $\mu_{1}$ by $\left(E_{1}-E_{12}\right)$, and decreases $\mu_{2}$ by $\left(E_{2}-E_{12}\right)$. The same energies accompany the opposite process. We call these processes internal charging.

Transport through the ring is possible when $\mu_{1}$ is on resonance with the reservoirs (i.e., when $\mu_{1}=\mu_{\text {res }}$ ). This resonance condition is irrespective of $\mu_{2}$. However, when $\mu_{2}$ is close to $\mu_{1}$ an electron can tunnel between the two Landau levels. This changes $\mu_{1}$ by an internal charging energy that switches the macroscopic conductance from on- to offresonance. We can use such switches in the conductance as a probe to detect tunnel events between the two confined Lan-

TABLE I. Summary of the device parameters. The number of electrons $N$ in a dot is estimated from the electron density $n_{s}$ in the bulk and the size of the dot. The filling factor $V_{\text {dot }}$ in the dot is estimated from the experiments in Sec. V.

\begin{tabular}{lccc}
\hline \hline Sample & Dot I & Dot II & Dot III \\
\hline Lithographic size & $0.8 \times 1.0 \mu \mathrm{m}^{2}$ & $0.6 \times 0.6 \mu \mathrm{m}^{2}$ & $0.8 \times 1.0 \mu \mathrm{m}^{2}$ \\
Number of electrons $(N)$ & $\approx 300$ & $\approx 200$ & $\approx 425$ \\
$n_{s}$ & $1.9 \times 10^{15} \mathrm{~m}^{-2}$ & $1.9 \times 10^{15} \mathrm{~m}^{-2}$ & $2.7 \times 10^{15} \mathrm{~m}^{-2}$ \\
Filling factor $2<\mu_{\text {dot }}<1$ & $\approx 3.4-6.8 \mathrm{~T}$ & $\approx 2.6-5.2 \mathrm{~T}$ & $\approx 4.2-8.4 \mathrm{~T}$ \\
\hline \hline
\end{tabular}




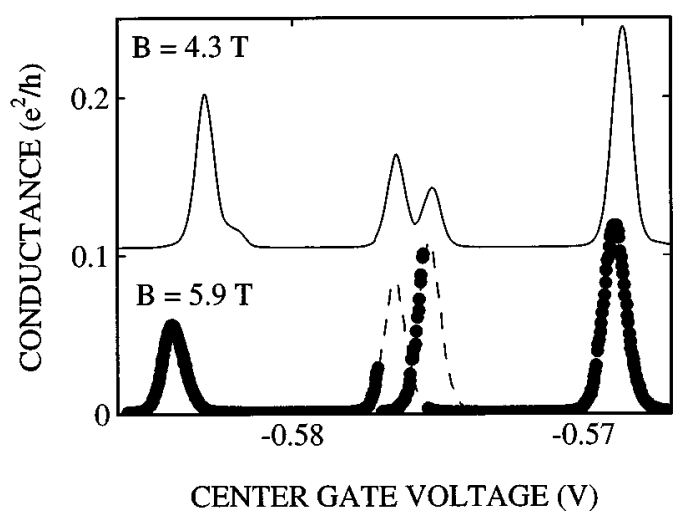

FIG. 3. Comparison between two traces of Coulomb oscillations measured in dot I at a field of $4.3 \mathrm{~T}$ (top curve) and 5.9 $\mathrm{T}$ (bottom curve). At low field, fast switching occurs that results in a continuous split peak, while at higher fields discrete switching is resolvable. The top curve has been shifted in gate voltage to facilitate the comparison.

dau levels. In particular, if the time between two switches is larger than the time constant of the measurement setup, it is possible to do a time-resolved measurement of singleelectron tunneling.

Note that the circuit of Fig. 1(b) resembles the electrometer-box configuration employed by Fulton, Gammel, and Dunkleberger ${ }^{14}$ and Lafarge et al., ${ }^{15}$ to measure single-electron hopping events in the superconducting and normal state of aluminum tunnel junctions. In our case, the barrier between the electrometer (the ring) and the box (the core) is not artificially fabricated, but results from the intrinsic properties of a two-dimensional electron gas in the quantum Hall regime.

\section{COULOMB OSCILLATIONS IN THE QUANTUM HALL EFFECT REGIME}

\section{A. Experiments}

At zero magnetic field, the dot can be described as one metallic region, weakly coupled to two electron reservoirs. The conductance through the dot at $B=0$ shows equally spaced Coulomb oscillations as a function of gate voltage, where each period corresponds to a change of one electron in the dot. ${ }^{16}$ When we apply a magnetic field perpendicular to the dot, we observe the conductance peaks shown in Fig. 2(b). This trace is taken at $4.3 \mathrm{~T}$, where only the two spinresolved states $\left(L L_{1}\right.$ and $\left.L L_{2}\right)$ of the lowest Landau level are occupied. A striking feature is that the trace shows a repeating pattern of a split oscillation alternated by three regular Coulomb oscillations. Furthermore, the peak spacing $\Delta V_{C}$ in Fig. 2(a) is not a constant as it is at $B=0$, but shows three different spacings: $1.5 \mathrm{mV}$ (between the two maxima of a split peak), $6.5 \mathrm{mV}$ (between a regular oscillation and a split peak), and $8 \mathrm{mV}$ (between two regular peaks).

The origin of the split peaks becomes clear at higher fields. Figure 3 shows a comparison between two traces of Coulomb oscillations measured at 4.3 and 5.9 T. The bottom curve shows that a split peak consists of two regular Coulomb peaks. To illustrate this we have added two dashed curves to each split peak (the dashed curves are taken from a

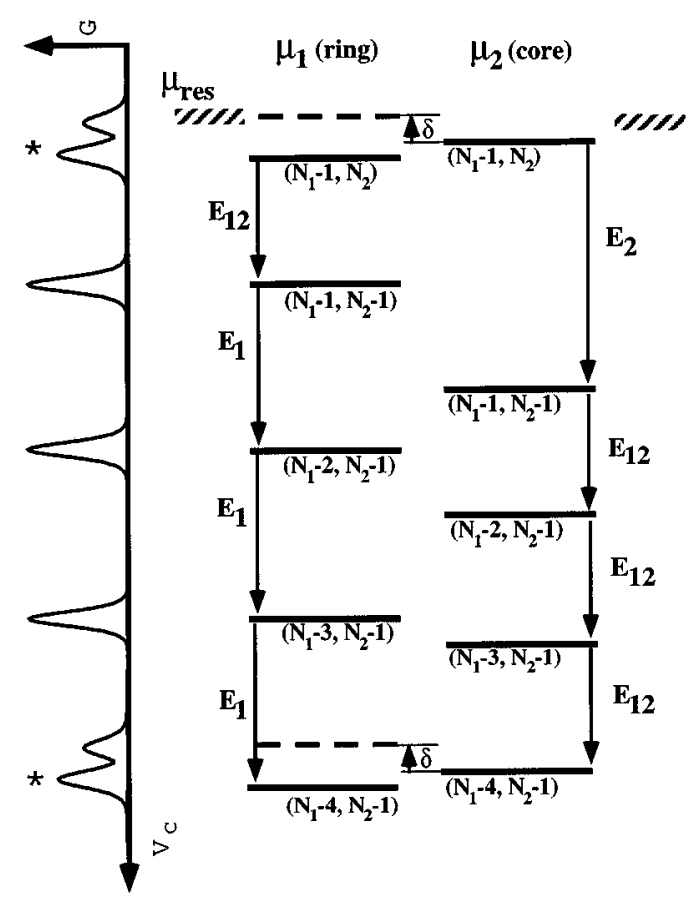

FIG. 4. Extension of Fig. 4 in Ref. 12. The left-hand side shows schematically the conductance $G$ when the gate voltage $V_{C}$ is varied. The spacings of the Coulomb oscillations are proportional to $E_{1}-E_{12}, E_{12}$, and $E_{1}$. Note that the special ratio of the energies $E_{1}, E_{2}$, and $E_{12}$ gives rise to three regular peaks in between two split peaks.

normal peak from which the height has been scaled ${ }^{17}$ ). In Ref. 12, we show that when the gate voltage is fixed and the conductance is measured versus time, it shows random switching between two discrete levels. We found that around $5.9 \mathrm{~T}$ the typical time between switches is about $200 \mathrm{sec}$. On the other hand, in the top curve measured in lower magnetic field, the typical time between switches is too short to be resolved by the time resolution of our measurement setup of typically $10 \mathrm{~ms}$. This smears out the switching and we observe a continuous split peak.

\section{B. Time-resolved tunneling of single electrons}

We now discuss that a switch in the conductance in Fig. 3 is a time-resolved measurement of a tunnel event by a single electron between the two Landau levels in the dot. As discussed in Sec. III, the current through the ring can be used as a probe to detect a tunnel event between the ring and the core. For a better understanding of these internal charging processes, we need to know the minimum energies to charge up the ring or the core by one electron.

The energy diagram of Fig. 4 in Ref. 12 can be used to illustrate the role of the internal charging energies. In that paper we show that the conductance is changed suddenly when an electron tunnels from $L L_{2}$ to $L L_{1}$. This singleelectron tunnel event switches the macroscopic conductance from off to on resonance. The conductance is switched back from on to off resonance when an electron tunnels back to $L L_{2}$. Depending on the energy difference $\delta$ between the initial and final state of the tunneling electron, these processes 
may require some assistance from fluctuating sources such as a finite temperature or noise in the environment.

We note that Fig. 4 in Ref. 12 shows only a few of the possible tunnel events between the core and the ring. The possible tunnel mechanisms depend on the precise values of the internal charging energies. Furthermore, we neglect cotunneling events, which can also provide an additional mechanism for changing the number of electrons in the ring and the core. The main conclusion, however, remains unaltered: $a$ switch in the conductance is the result of the timeresolved transfer of a single electron between the ring and the core.

\section{Experimental determination of the internal charging energies}

The three charging energies can be obtained from the measured peak spacings in Fig. 2(a). Unequal internal charging energies $E_{1}$ and $E_{12}$ result in unequally spaced Coulomb oscillations. We now discuss that for special values of the charging energies a periodic pattern of split and regular peaks can be observed.

Figure 4 of the present paper shows an extension of Fig. 4 in Ref. 12. The electrochemical potentials $\mu_{1}$ and $\mu_{2}$ denote the minimum energy for having a certain number of electrons in the ring $\left(L L_{1}\right)$ and the core $\left(L L_{2}\right)$. The left-hand side shows schematically the conductance through the dot versus the center-gate voltage $V_{C}$. By decreasing the gate voltage $V_{C}$ one electron is removed from $L L_{2} . \mu_{2}$ then drops by $E_{2}$ and $\mu_{1}$ by $E_{12}$. The next three conductance peaks are again regular. The energy difference $\delta$ between the initial and final state of a tunneling electron is too large for these three peaks. Each time when a regular peak occurs $\mu_{1}$ drops by $E_{1}$ and $\mu_{2}$ by $E_{12}$. Since $E_{1}>E_{12}$ the energy difference $\delta$ becomes smaller after a regular peak. In the last oscillation denoted by an asterisk, $\delta$ is small enough to get internal charging processes and here the conductance shows a split peak.

This diagram shows that there are three different peak spacings in gate voltage. These are proportional to $E_{1}-E_{12}, E_{12}$, and $E_{1}$. This is in agreement with the experiments. In Fig. 2(a) we found three peak spacings: 1.5 (between the two maxima of a split peak), 6.5 , and $8 \mathrm{mV}$. Using a factor of 25 for this sample to convert the gate voltage scale to energy, ${ }^{18}$ we find $E_{1}-E_{12}=60 \mu \mathrm{eV}$, $E_{12}=260 \mu \mathrm{eV}$, and $E_{1}=320 \mu \mathrm{eV}$. Note that these three values are consistent, although, they are determined independently. From additional experiments we find that the splitting of the peaks starts to smear out for bias voltages between 60 and $100 \mu \mathrm{V}$. This is also in good agreement with the estimated energy separation associated with a split peak $E_{1}-E_{12}=60 \mu \mathrm{eV}$.

The value of $E_{2}$ can be found from the pattern of split and regular peaks. In Fig. 2 every split peak is alternated by three regular peaks. Figure 4 illustrates that this occurs when the ratio of the three charging energies $E_{1}, E_{2}$, and $E_{12}$ has a special value. These ratios can be found by considering that the energy difference $\delta\left(=\mu_{1}-\mu_{2}\right)$ is the same for the top and bottom oscillation. Summing up the energy changes of $\mu_{1}$ and $\mu_{2}$ from top to bottom, gives the relation

$$
p\left(E_{1}-E_{12}\right)=E_{2}-E_{12},
$$

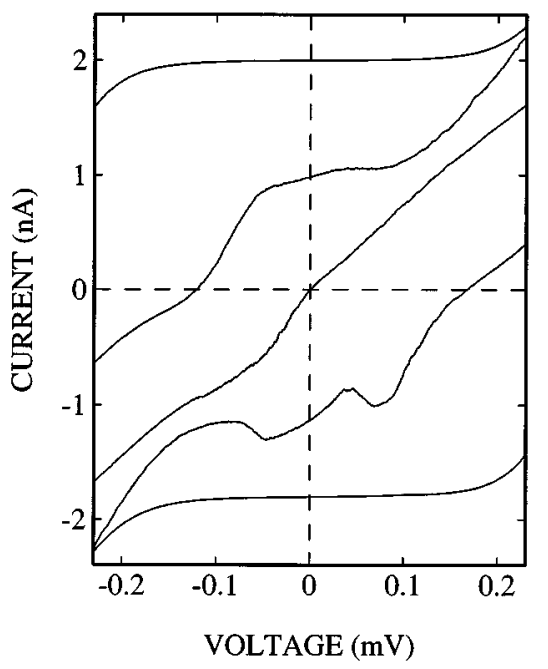

FIG. 5. Current-voltage $(I-V)$ characteristics for five different center gate voltages at $4.6 \mathrm{~T}$. From top to bottom $V_{C}=-585,-582,-581,-580$, and $-578 \mathrm{mV}$. The curves are offset for clarity $(I=0$ occurs at $V=0)$. Measurements are performed on dot III.

where $p$ denotes the number of regular peaks between two split peaks. In Fig. 2 we find $p=3$, while for Fig. 2 in Ref. 12 we find $p=3$ or 4 . Using $p=3.5$ yields $E_{2}=460 \mu \mathrm{eV}$. The fact that $E_{2}>E_{1}$ is consistent with the core being smaller than the ring. In previous work we showed that $p$ can be tuned from 2 to 4 by increasing the magnetic field. ${ }^{19}$ To explain this effect one can parametrize the internal charging energies by capacitances between the metallic parts and their surroundings. ${ }^{16,10,20}$ The total capacitances between the gates and $L L_{1}$ and $L L_{2}$ are defined as $C_{1}$ and $C_{2}$, and the inter$L L$ capacitance as $C_{12}$ (see Fig. 1). Using the expressions of Ref. 6 for $E_{1}, E_{2}$, and $E_{12}$ yields $p=C_{1} / C_{2}$. The area of the $L L_{1}$ ring increases with magnetic field and the area of $L L_{2}$ decreases. ${ }^{8}$ This changes the capacitances and leads to an increasing ratio $p=C_{1} / C_{2}$ with magnetic field. An alternative way to interpret this result is that at high magnetic fields the number of electrons in $L L_{1}$ is larger than at low fields leading to more regular than split peaks. A similar effect has been reported by Heinzel et al. ${ }^{21}$

The charging energies can also be obtained from measurements at finite source-drain voltages. Figure 5 shows five current-voltage $(I-V)$ characteristics measured for different center gate voltages. The data are taken at $4.6 \mathrm{~T}$ in the fastswitching regime (see the bottom curve of Fig. 2 in Ref. 12). In the top $I-V$ curve, the current is suppressed around $V=0$ due to the Coulomb blockade. From the width of the current gap we get $E_{1} \approx 350 \mu \mathrm{eV}$, in reasonable agreement with the value determined above. When the gate voltage is changed from the top to bottom curve, the Coulomb gap reduces. In the center curve the gap is tuned away. The conductivity is finite around $V=0$, which corresponds to a peak in the conductance in Fig. 3(b). When $V_{C}$ is decreased further, the $I-V$ shows a negative differential conductance (NDC) at $V= \pm 80 \mu \mathrm{V}$. In the bottom curve, the maximum Coulomb gap is recovered. From top to bottom, the gate voltage is changed by $\Delta V_{C}=7 \mathrm{mV}$, which is the period of the Coulomb oscillations in this sample. 
The NDC is caused by the transfer of an electron between the ring and the core. Around $V= \pm 80 \mu \mathrm{V}$, the current drops as the bias voltage is increased. At these values it is favorable to redistribute the charge in the dot. This is caused by a difference in the effective capacitances between the ring and the leads, and the core and the leads. Increasing $V$ changes $\mu_{1}$ and $\mu_{2}$ in a different way, thereby changing the energy difference $\delta=\mu_{1}-\mu_{2}$. This can result in the transfer of an electron from the ring to the core or vice versa depending on the value of the inter Landau-level capacitance, the magnetic field and the exact voltage drops across the two QPC barriers. In the time-resolved regime we observed a similar behavior, but in this case the transfer of an electron results in discrete switching between "two $I$ - $V$ curves" (not shown).

\section{Calculation of the conductance through the dot}

Once the values of the three charging energies are known, it is easy to calculate the positions where the conductance peaks occur. The electrochemical potentials of the ring and the core are given by ${ }^{7}$

$$
\begin{aligned}
& \mu_{1}\left(N_{1}, N_{2}, V_{C}\right)=\varepsilon_{1}+\left(N_{1}-\frac{1}{2}\right) E_{1}+N_{2} E_{12}+e \alpha_{1} V_{C} \\
& \mu_{2}\left(N_{1}, N_{2}, V_{C}\right)=\varepsilon_{2}+\left(N_{2}-\frac{1}{2}\right) E_{2}+N_{1} E_{12}+e \alpha_{2} V_{C}
\end{aligned}
$$

We ignore terms depending on magnetic field and also neglect the capacitance between the dot and the reservoirs. $\varepsilon_{1}$ and $\varepsilon_{2}$ denote the highest-occupied single-particle energy of the electrons in $L L_{1}$ and $L L_{2}$. The values $\alpha_{1}$ and $\alpha_{2}$ depend on the gate capacitance and determine at which rate $\mu_{1}$ and $\mu_{2}$ change as a function of the center gate voltage. Assuming that $\alpha_{1}$ and $\alpha_{2}$ are equal gives $\alpha_{1}=\alpha_{2}=25$. From Eqs. (2) and (3) and the charging energies determined above, we can calculate $\mu_{1}\left(N_{1}, N_{2}, V_{C}\right)$.

The conductance $G$ through $L L_{1}$ is determined by the energy difference $\left(\mu_{\text {res }}-\mu_{1}\right)$. The line shape of a Coulomb peak is given by ${ }^{22}$

$$
\frac{G\left(\mu_{1}\right)}{G_{\max }}=\cosh ^{2}\left[\left(\mu_{1}-\mu_{\mathrm{res}}\right) / 2.5 k_{B} T\right] .
$$

$G_{\max }$ is the maximum peak conductance. This expression assumes that for a given gate voltage $V_{C}$ the electrochemical potential $\mu_{1}$ is constant. For a dot with two Landau levels this is not generally true since an internal charging process changes $\mu_{1}$. To include this we use a simple model. From Eqs. (2) and (3) we determine $N_{1}$ and $N_{2}$ in the ground state at a given gate voltage. Besides the conductance via the ground state $\mu_{1}\left(N_{1}, N_{2}\right)$ [i.e., transport via the states $\left.\left(N_{1}, N_{2}\right) \leftrightarrow\left(N_{1}-1, N_{2}\right)\right]$, it is also possible to conduct via a nearby charge state. For instance, if an electron tunnels from $L L_{2}$ to $L L_{1}$ then transport may take place via $\mu_{1}\left(N_{1}+1, N_{2}-1\right)$ [i.e., via the states $\left(N_{1}+1, N_{2}-1\right)$ $\leftrightarrow\left(N_{1}, N_{2}-1\right.$. Taking $P_{1}$ as the probability that transport takes place via $\mu_{1}\left(N_{1}+1, N_{2}-1\right)$, and $P_{2}$ as the probability that transport takes place via $\mu_{1}\left(N_{1}, N_{2}\right)$, we obtain

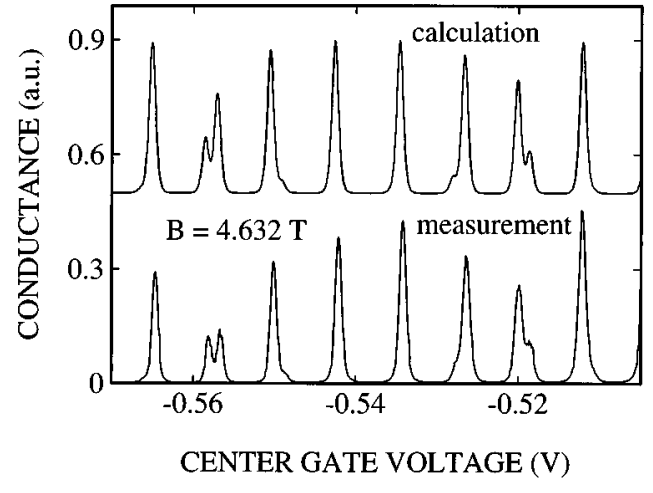

FIG. 6. Comparison of a measured trace to a calculation of the conductance through the dot using Eqs. (4)-(6). The parameters are $T=120 \mathrm{mK}, E_{1}=320 \mu \mathrm{eV}, E_{2}=460 \mu \mathrm{eV}$, and $E_{12}=60 \mu \mathrm{eV}$.

$$
\begin{aligned}
\frac{G\left(V_{C}\right)}{G_{\max }}= & G\left(\mu_{1}\left(N_{1}, N_{2}, V_{C}\right)\right) P_{2} \\
& +G\left(\mu_{1}\left(N_{1}+1, N_{2}-1, V_{C}\right)\right) P_{1} .
\end{aligned}
$$

The probability that an electron tunnels between the ring and the core depends on the energy difference $\delta$ between the initial and final state of the electron, which can be calculated from Eqs. (2) and (3). If we assume that these transitions are thermally activated, and thus neglect noise from the environment, then the ratio $P_{1} / P_{2}$ is given by a Boltzmann factor:

$$
\frac{P_{1}}{P_{2}}=\exp \left(\frac{\delta}{k_{B} t}\right) \text {. }
$$

From Eqs. (2)-(6) and the experimental charging energies, we have calculated the conductance for each $V_{C}$. Using a temperature $T=120 \mathrm{mK}$, we find excellent agreement between experiment and calculation (see Fig. 6). The positions of the conductance peaks, including the pattern of split and regular peaks, are reproduced in detail in the calculation. This shows that Eqs. (2) and (3) describe the charge states of a dot with two Landau levels very well. Remarkably, this simple calculation reproduces both the experimentally observed periodic pattern and amplitude of the split peaks. Below, we show data that confirm the validity of Eq. (6).

\section{MAGNETIC-FIELD DEPENDENCE OF THE INTERNAL CHARGING PROCESSES}

\section{A. Magneto-Coulomb oscillations and single-charge depopulation}

The number of electrons in each $L L$ is determined by the Landau-level degeneracy and the confinement potential of the dot. Each time a flux quantum is added to the dot, $L L_{1}$ can accommodate an extra electron. This results in the transfer of an electron from $L L_{2}$ to $L L_{1}$, while keeping the total number of electrons in the dot constant. An understanding of this process can be obtained in terms of energy. Increasing the magnetic field changes the self-consistent charge distribution in the dot: the real Landau-level degeneracy increases, which causes charge in $L L_{1}$ to flow towards the center of the dot. This change in the electron distribution decreases $\mu_{1}$ relative to $\mu_{2}$, which continues until it is energetically favor- 


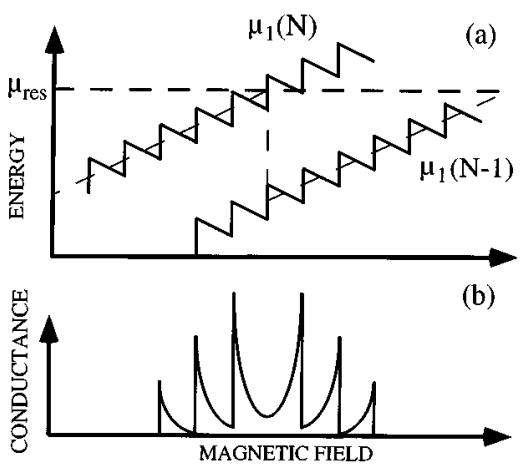

FIG. 7. (a) Sawtooth-shaped oscillations of $\mu_{1}$ versus magnetic field for $N$ and $N-1$ electrons in the dot. (b) Effect of the oscillating electrochemical potential $\mu_{1}$ on the conductance through the dot. Note the reversing of the sawtooths on the left- and right-hand sides and that the shape of the magnetoconductance resembles the shape of a "tree."

able to transfer one electron from $L L_{2}$ to $L L_{1} \cdot{ }^{6,7}$ As a function of magnetic field $\mu_{1}$ changes in a sawtooth manner [see Fig. $7(\mathrm{a})]$. The steep rises of $\mu_{1}$ occur when an electron tunnels from $L L_{2}$ to $L L_{1}$. The overall linear increase of $\mu_{1}$ (dashed line) is mainly caused by the increase of the cyclotron energy $\hbar \omega_{c}$.

Figure 7(b) illustrates the effect of a sawtooth-shaped $\mu_{1}$ on the conductance. ${ }^{23}$ If $\mu_{1}(N)<\mu_{\text {res }}$ (low-field side) the conductance $G$ in Fig. 10(b) rises very steeply when an electron tunnels from $L L_{2}$ to $L L_{1}\left[\mu_{1}(N)\right.$ jumps towards $\left.\mu_{\text {res }}\right]$. The conductance $G$ decreases slowly when the magnetic field is increased further until the next electron can be transferred from $L L_{2}$ to $L L_{1}$. On the other hand, when $\mu_{1}(N)>\mu_{\text {res }}$ the conductance decreases when an electron is transferred from $L L_{2}$ to $L L_{1}\left[\mu_{1}(N)\right.$ jumps away from $\left.\mu_{\text {res }}\right]$ and increases slowly when the magnetic field is increased further. Hence, we expect that the orientation of the sawtooths reverses at the center of the resonance. ${ }^{23}$ Figure 7(b) shows that a magneto-Coulomb oscillation has the shape of a "tree.' The envelope of the tree corresponds to a change in the total number of electrons in the dot by one and can be interpreted as a magneto-Coulomb oscillation. ${ }^{23,24}$

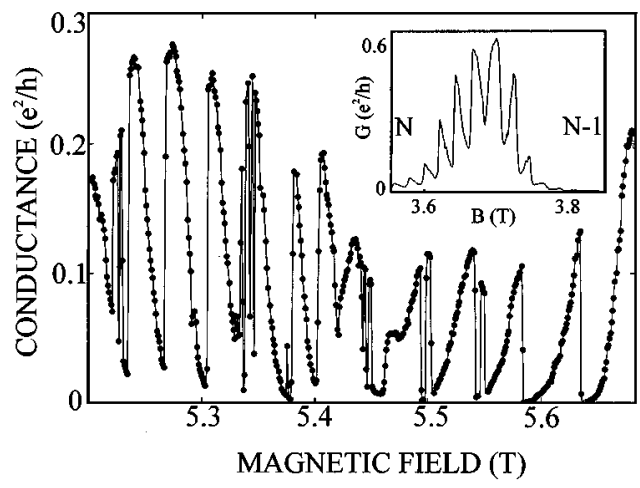

FIG. 8. Conductance through the dot as a function of the magnetic field using a time constant of $500 \mathrm{~ms}$, illustrating that the frequency of switching decreases with the field. The inset shows the conductance $G$ versus the magnetic field $B$ in a lower-field regime, where the switches are too fast to be resolved. Measurements are performed on dot I.

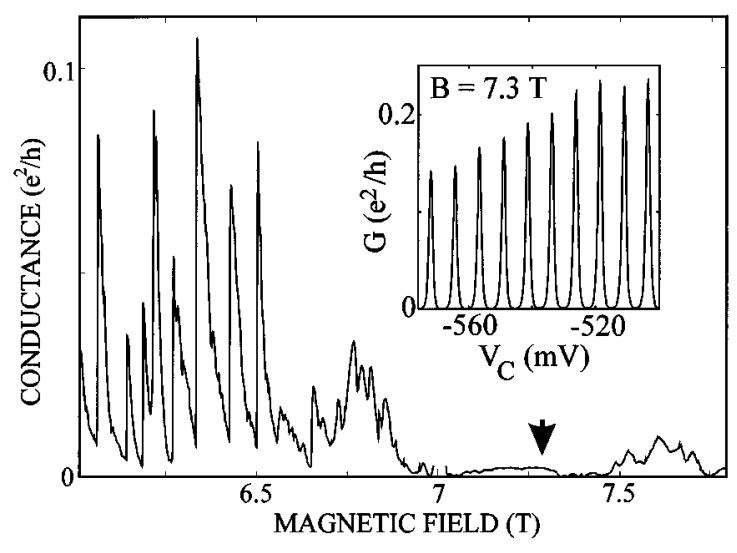

FIG. 9. Conductance through the dot as a function of the magnetic field. Around 6.6 T, the sawtooths disappear, which indicates that the core is completely depopulated. This is supported by the inset, which shows only regular Coulomb blockade oscillations at a field of $7.3 \mathrm{~T}$. Measurements are performed on dot I.

This simple picture is sufficient to describe our experimental observations. The inset of Fig. 8 shows the conductance through the dot versus magnetic field in the low-field regime, where we observed the continuous split peaks of Fig. 2(b). The conductance shows one large oscillation with small sawtoothed oscillations superimposed on it. The large oscillation is an observation of a magneto-Coulomb oscillation. The orientation of the small sawtooth oscillations changes as expected. The period of the small sawtooths is $23 \mathrm{mT}$, which corresponds to adding an extra flux quantum $h / e$ to a disk with a diameter of $480 \mathrm{~nm}$. This is within $20 \%$ of the estimated dimensions of the dot. The shape and the period of the sawtooths show that each period corresponds to the transfer of one electron from the core to the ring. This means that by sweeping the magnetic field we can monitor the singlecharge depopulation of the core. ${ }^{25}$

\section{B. Magnetic-field dependence of the switching rate}

\section{Switching on the scale of several flux quanta}

Figure 8 shows the conductance in a higher, time-resolved field regime. Around 5.5 $\mathrm{T}$ the sawtooths again clearly

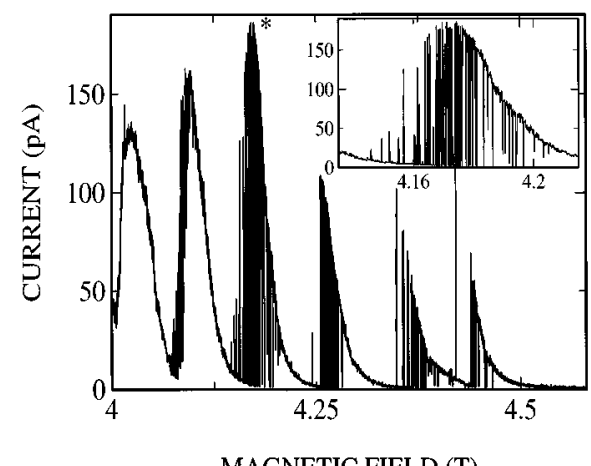

FIG. 10. Conductance through the dot as a function of the magnetic field measured in sample II. The size of this dot is about a factor of 2 smaller than dot I. The regime $2<V_{\text {dot }}<1$ therefore occurs at a lower magnetic field. Inset: enlargement of the sawtooth denoted by an asterisk. 
change orientation. In the inset, we see continuous transitions between the sawtooths, while in the main plot discrete transitions occur. Note that multiple switching occurs at some magnetic fields and that the switching rate decreases with magnetic field. Around $5.35 \mathrm{~T}$ frequent switching occurs, while around $5.5 \mathrm{~T}$ only one or two switches are observed. This behavior is consistent with the transition of continuous split peaks at low field to split peaks with discrete switching at higher fields. Note also that the measured switching rate for the split peaks agrees with the rate observed in the sawtooths. The only difference is that as a function of gate voltage switching occurs between two branches of a split peak, while versus magnetic field switching occurs between two sawtooths.

In Fig. 9, at even higher fields, the switching rate is very low. Now only one switch between two sawtooths occurs and the period of the sawtooths becomes irregular. Due to the large time scales we are not always probing the ground state. Around 6.6 T the sawtooths disappear. Around this magnetic field presumably all electrons in the core are transferred to $L L_{1}$. The dot can now be considered as one metallic disk. This is supported by the regular Coulomb oscillations as a function of the center gate voltage at a field of $7.3 \mathrm{~T}$ in the inset. The period is constant and switching does not occur anymore.

Counting the number of sawtooths as a function of magnetic field gives an estimate of the total number of electrons in the core. In the field interval $3.3-6.6 \mathrm{~T}$ we observed 104 oscillations, which corresponds to having $N_{2}=104$ electrons in the core and roughly $N=210$ electrons in the dot at $3.3 \mathrm{~T}$. This is in reasonable agreement with the size and the electron density of the dot (see Table I).

Although, we do not observe any switching in this sample at fields larger than $6.6 \mathrm{~T}$, there is still some structure visible in the conductance around $6.8 \mathrm{~T}$. This might be related to the last few electrons being removed from the core. ${ }^{26}$ Another possibility is that correlations in the fractional quantum Hall effect (FQHE) regime start to play a role. In the FQHE regime $L L_{1}$ may split up in separate fractional edge channels. ${ }^{27,28}$ Similar switching as we have described in this paper may also be observable at higher magnetic fields and can hopefully be used to clarify the internal structure of a Coulomb island in the FQHE regime.

\section{Switching on the scale of the one flux quantum}

Switching occurs periodically in adding an extra flux quantum to the dot. However, switching within one sawtooth is only possible in a very small field interval. We have studied this dependence in more detail in sample II. Figure 10 shows six sawtooths with a period of roughly $80 \mathrm{mT}$. This corresponds to threading an extra-flux quantum through a disk with a diameter of $260 \mathrm{~nm}$, which is close to the estimated dot size (see Table I). Note that also in this smaller sample the switching rate decreases with field.

Besides this change in switching rate on the scale of a few flux quanta, there is also a change in switching rate within one flux quantum. In the inset of Fig. 10 we have enlarged one of the sawtooths from the main plot. At the low field side no switches are observed. When the field is increased to $B \approx 4.15 \mathrm{~T}$ switching starts to occur. Increasing the field further increases the switching rate and around $B \approx 4.17 \mathrm{~T}$ the

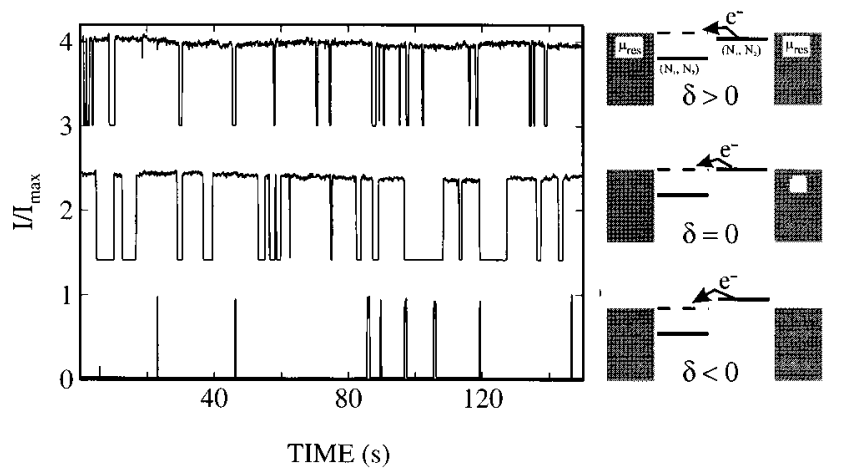

FIG. 11. Left-hand side: conductance of dot II versus time for three different magnetic fields (from top to bottom: 4.428, 14.417, $4.405 \mathrm{~T}$ ). Measurements are performed on dot II. Right-hand side: energy diagrams for three different magnetic fields showing $\mu_{1}$ of $\mathrm{LL}_{1}$ and $\mu_{2}$ of $\mathrm{LL}_{2}$.

conductance is an equal amount of time in the "up" and "down" state. Continuing to increase the field causes the switching rate to decrease again and now the conductance is mainly in the up state.

This tendency is clearly visible when we fix the magnetic field and measure the conductance through the dot versus time. Figure 11 shows time traces for three different magnetic fields. In the bottom curve, the conductance is mainly in the down state $\left(\tau_{\text {up }} \ll \tau_{\text {down }}\right)$, with random switches to the up state. In the middle curve, the average up and down time are the same $\left(\tau_{\text {up }} \approx \tau_{\text {down }}\right)$, while in the top curve the conductance is mainly in the up state $\left(\tau_{\text {up }} \gg \tau_{\text {down }}\right)$.

The magnetic-field dependence of $\mu_{1}$ and $\mu_{2}$ is responsible for this effect. In Sec. V A we already discussed the magnetic-field dependence of the energy difference $\delta$. The right-hand side of Fig. 11 illustrates the effect of a change in $\delta$ on the switching rate. In the topmost diagram an electron needs to overcome an energy $\delta$ to tunnel from the core to the ring $(\delta<0)$. The conductance will therefore be mainly in the down state. Upon increasing the field $\delta$ decreases until $\delta=0$ and now the average up and down time is the same. A further increase makes $\delta>0$ and now the conductance will be mainly in the up state. Figure 11 is a direct measurement of the rate for single-electron tunneling from the ring to core for a given energy difference $\delta$.

We determined the average values of $\tau_{\text {up }}$ and $\tau_{\text {down }}$ as a function of magnetic field by averaging over more than 500 switches. Figure 12(a) shows a plot of the ratio $\tau_{\text {up }} / \tau_{\text {down }}$ measured within one sawtooth. This ratio clearly exhibits an exponential over more than four decades. It can therefore be described by a formula like

$$
\frac{\tau_{\text {up }}}{\tau_{\text {down }}}=\exp \left(\frac{\gamma\left(B-B_{0}\right)}{k_{B} T}\right) .
$$

At $B=B_{0}$, the average up and down times are equal $\left[B_{0}=4.693 \mathrm{~T}\right.$ in Fig. 12(a)]. Note that the ratio $\left(\tau_{\text {up }} / \tau_{\text {down }}\right)$ equals $P_{1} / P_{2}=\exp \left(\delta / k_{B} T\right)$, which gives $\delta=\gamma\left(B-B_{0}\right)$. An estimate for $\delta$ can be obtained from the slope in Fig. 12(a). Using a temperature $T=100 \mathrm{mK}$ yields $\gamma=2.2 \mu \mathrm{eV} / \mathrm{mT}$. This means that in one period $\Delta B(=70 \mathrm{mT})$ of the sawtooths in Fig. 10, $\delta$ changes by $\Delta B \gamma=150 \mu \mathrm{eV}$. Another 

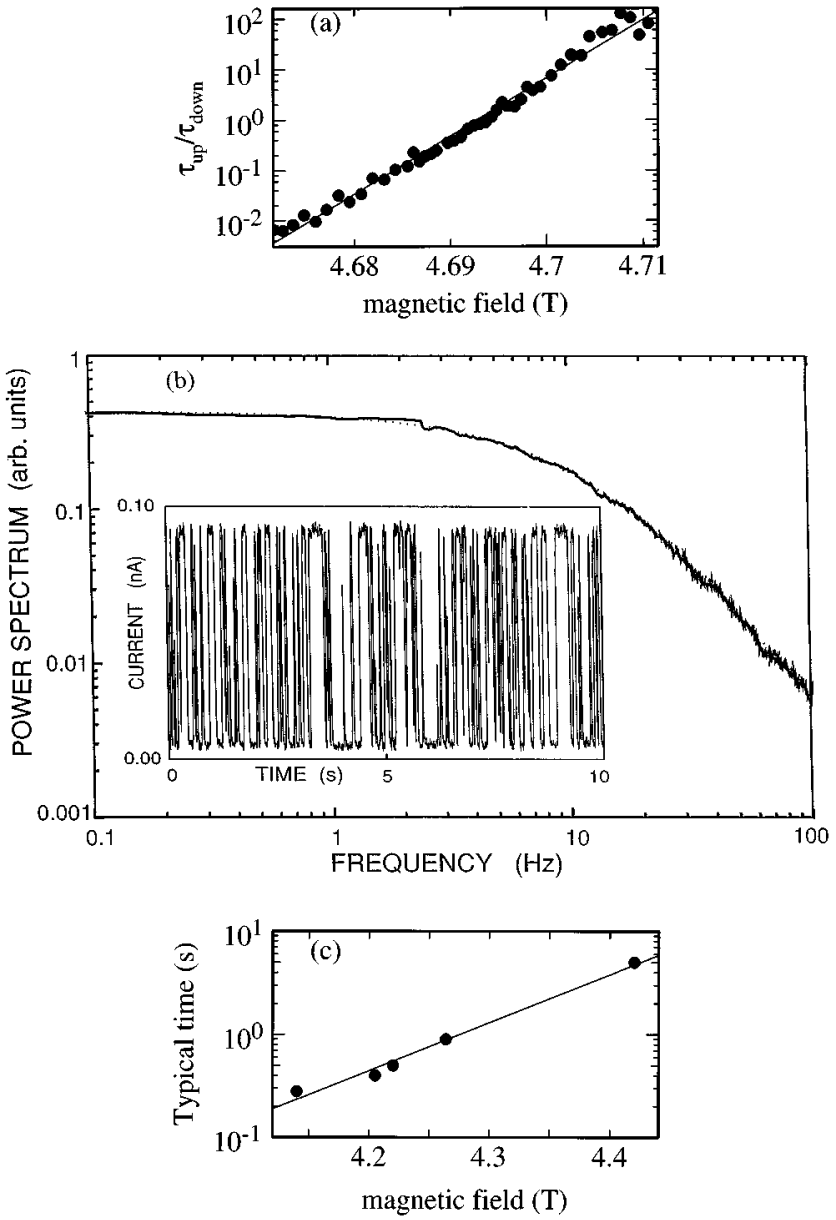

FIG. 12. (a) Ratio of the average up and down times $\tau_{\text {up }} / \tau_{\text {down }}$ versus the magnetic field. Measurements are performed on dot II. (b) Power spectrum of the switch signal in the inset. (c) Typical switching time $\left(\tau_{\text {up }}=\tau_{\text {down }}\right)$ versus the magnetic field.

estimate for this number can be obtained from Fig. 4. The period of a sawtooth corresponds to an energy change in $\delta$ by $E_{1}+E_{2}-2 E_{12}$. The value of $150 \mu \mathrm{eV}$ is in reasonable agreement with the value we found from $E_{1}, E_{2}$, and $E_{12}$ in this sample.

The magnetic-field dependence of the probability to tunnel between two Landau levels manifest itself in a different way in the lower field regime. For instance, the evolution of a split peak into a regular peak in Fig. 2 of Ref. 12 is caused by this effect. The individual switches are not resolved in this field regime, but they do influence the time-averaged current through the dot. The two branches of the split peak in the bottom curve of Fig. 2 of Ref. 12 (denoted by an asterisk) have the same amplitude. However, when the field is increased the "up and down times" change, causing a lower amplitude of the left peak and an increase of the right split peak. This continues and the split peaks become regular again. When the field is increased by one flux quantum the bottom curve resembles the top curve in Fig. 2 of Ref. 12, since the ratio $\left(\tau_{\text {up }} / \tau_{\text {down }}\right)$ is periodic in threading an extra flux quantum through the dot.

\section{Two-level fluctuator}

The concept of single-electron tunneling between the Landau levels provides a quantitative understanding of both the discrete conductance switches and the conductance in the low-field regime. The measurements in this section show that the dot can be considered as a completely controllable twolevel fluctuator. The probability to be in the up or down state is determined by $\delta$. This energy can be tuned continuously by the magnetic field and becomes zero periodically in adding a flux quantum to the dot.

The statistics of internal switching processes can be determined by plotting the power spectrum of a switch signal versus frequency. Figure 12(b) shows the power spectrum of the time trace in the inset. The dashed line is a Lorentzian fit. The excellent agreement implies that switching between the up and down state occurs randomly. ${ }^{29}$

We already mentioned that the typical switch time $\left(\tau_{\text {up }}=\tau_{\text {down }}\right)$ increases with every added flux quantum. In Fig. 12(c) we have plotted this time versus magnetic field. This plot was constructed by measuring data sets like in Fig. 12(a). Using these data we determined at which magnetic field $\left(\tau_{\text {up }} / \tau_{\text {down }}\right)=1$ occurs. The relation between the switching rate and magnetic field clearly exhibits an exponential behavior.

\section{Period of the magneto-Coulomb oscillations}

In this section we discuss the magneto-Coulomb oscillations in more detail. We show that the oscillations are periodic in unit charge onto the dot. In addition, we see signatures that near filling factor 2 in the bulk the charge distribution over Landau levels in the reservoirs strongly affects the period of the magneto-Coulomb oscillations. The charge distribution in the reservoirs also seems to be responsible for the remarkable hysteresis.

Figure 2(a) in Ref. 23 shows the magnetoconductance of dot III for different center gate voltages. In the bottom curve, we distinguish three different regions. For $B<5.4 \mathrm{~T}$ a superposition of two types of oscillations are visible: one with a large period of about $500 \mathrm{mT}$ and another with a smaller period of $25 \mathrm{mT}$. Around $5.6 \mathrm{~T}$ the shape of the oscillations changes markedly, becoming sharply peaked. We argue below that this can be attributed to the rapid depopulation of the topmost spin-split $L L$ in the reservoirs. For $B>5.75 \mathrm{~T}$, we see small oscillations with a period of about $33 \mathrm{mT}$ superimposed on a varying background

The large oscillations for $B<5.4 \mathrm{~T}$ with a period of roughly $500 \mathrm{mT}$ are magneto-Coulomb oscillations. This is demonstrated by two experimental observations. Increasing the gate voltage shifts the magneto-Coulomb oscillations to a larger field value. From bottom to top in Fig. 2(a) in Ref. 23, the gate voltage is changed by $4.5 \mathrm{mV}$, which shifts the magneto-Coulomb oscillations by one period. This gate voltage change is exactly the measured period of the Coulomb oscillation in this sample. Therefore, each period of the magneto-Coulomb oscillations corresponds to a change of one electron in the dot. This implies that the period of 500 $\mathrm{mT}$ should correspond to a change in the electrochemical potential $\mu_{1}$ by the charging energy $E_{1}$. Roughly, $\mu_{1}$ follows the Landau-level energy $\left(\frac{1}{2}\right) \hbar \omega_{c}$, where $\hbar \omega_{c}$ is the cyclotron energy (we neglect the much smaller Zeeman energy). Over $500 \mathrm{mT}, \frac{1}{2} \hbar \omega_{c}$ changes by $430 \mu \mathrm{eV}$. This is indeed close to the value we found for the charging energy $E_{1}=320 \mu \mathrm{eV}$ in this sample. 
Such a simple evaluation does not work around 5.6 T. In this field range the magneto-Coulomb oscillations have a much smaller period of about $100 \mathrm{mT}$. A Hall measurement of the bulk 2DEG reservoirs shows that the filling factor of the reservoirs approaches $\nu=2$ around 5.6 T. The third Landau level in the reservoirs is then being emptied. Until now we have ignored the influence of the reservoirs on the charge states in the dot. We now show that including the charge distribution in the reservoirs can explain in detail the anomalous magneto-Coulomb oscillations around 5.6 T.

In a ballistic sample, where localized states are absent, the topmost Landau level ( $n=1$, the lowest spin state) suddenly depopulates on approaching $\nu=2$. This leaves only the two spin states for $n=0$ filled. In the self-consistent edge picture ${ }^{8}$ this implies a change in the shape of the electrostatic potential at the edge. A changing edge potential just outside the dot induces a changing electrostatic potential in the dot. Note that we do no invoke any variations in the electrochemical potentials in the reservoirs. All leads are connected directly, or via voltage sources, to ground.

In the self-consistent picture emptying the topmost Landau level means that the edge of its compressible region moves from the sample edge into the bulk. Simultaneously, its electrons are transferred to the lower Landau levels. This changes the electrochemical potentials of the dot by $\beta_{1} \hbar \omega_{c}$ and $\beta_{2} \hbar \omega_{c}$. The coefficient $\beta_{1}=C_{1, \text { res }} / C_{1}$ and $\beta_{2}=C_{2 \text {,res }} / C_{2}$ with $C_{1 \text { res }}$ and $C_{2 \text { res }}$ representing the capacitive coupling between the compressible regions in the reservoirs and the compressible regions of $L L_{1}$ and $L L_{2}$ in the dot. Since the boundary of the former regions are changing when crossing an integer filling factor the coupling $\beta_{1,2}=\beta_{1,2}(B)$ has a magnetic-field dependence. In fact, this field dependence is similar to the sawtooth oscillations in the Fermi energy (i.e., chemical potential) when Landau levels are being depopulated. Including this magnetic contribution in Eqs. (2) and (3), together with the increase of the cyclotron and spin energy of the electrons in the dot, yields ${ }^{30}$

$$
\begin{aligned}
\mu_{1}\left(N_{1}, N_{2}, V_{c}\right)= & \varepsilon_{1}+\left(N_{1}-\frac{1}{2}-n_{1}\right) E_{1}+\left(N_{2}-n_{2}\right) E_{12} \\
& +e \alpha_{1} V_{C}+\frac{1}{2} \hbar \omega_{c}-g \mu_{B} B+\beta_{1} \hbar \omega_{c}, \\
\mu_{2}\left(N_{1}, N_{2}, V_{C}\right)= & \varepsilon_{2}+\left(N_{2}-\frac{1}{2}-n_{2}\right) E_{2}+\left(N_{1}-n_{1}\right) E_{12} \\
& +e \alpha_{2} V_{C}+\frac{1}{2} \hbar \omega_{c}+g \mu_{B} B+\beta_{2} \hbar \omega_{c} .
\end{aligned}
$$

$N_{1}$ and $N_{2}$ can only change by an integer; $n_{1}$ and $n_{2}$, however, can change continuously. For a parabolic confinement potential, the change in the self-consistent electron distribution with magnetic field can be modeled by ${ }^{6}$

$$
\begin{gathered}
n_{1}=N\left(\frac{\beta}{2} \frac{e B}{n_{\mathrm{dot}} h}-\frac{1}{2} \frac{(e B)^{3}}{\left(n_{\mathrm{dot}} h\right)^{3}}\right), \\
n_{2}=N-n_{1},
\end{gathered}
$$

where $N=N_{1}+N_{2}$ is the total number of electrons in the dot and $n_{\mathrm{dot}}$ indicates the electron density in the center of the dot.

Figure 13 shows the result of the calculated conductance versus field for increasing $\beta$ 's, i.e., for increasing capaci-

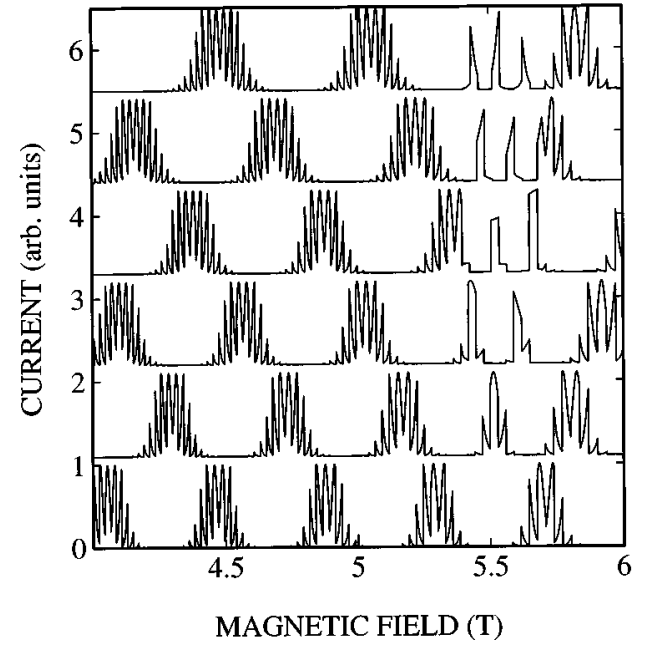

FIG. 13. Calculation of the conductance through the dot using Eqs. (9) and (10). The coupling between the dot and the reservoirs has been increased from zero (bottom curve) to $\beta_{1}=\beta_{2}=0.1$ (top curve) in increments of 0.2 . The parameters we used for the calculation are $n_{\mathrm{dot}}=1.92 \times 10^{15} \mathrm{~m}^{-2}, N=300, E_{1}=0.4 \mu \mathrm{eV}$, $E_{2}=0.6 \mu \mathrm{eV}, E_{12}=0.32 \mu \mathrm{eV}, g=2$, and $T=150 \mathrm{mK}$.

tance between dot and reservoirs. The calculation is similar as in Sec. IV, but now using Eqs. (8)-(10). We assume symmetric couplings to the leads, i.e., $\beta_{1}=\beta_{2}=\beta$. We let $n$ go from 1 to 0 around $5.6 \mathrm{~T}$, as determined from the Hall conductance of the 2DEG reservoirs (we ignore the small spin contribution to the changes in the reservoirs). The transition $n=1$ to $n=0$ has a finite width due to disorder in the reservoirs. We include this by letting $\beta(B)$ change linearly from a given value at $5.45 \mathrm{~T}$ to zero at 5.75 .

The bottom curve corresponds to $\beta=0$. This curve shows equidistant magneto-Coulomb oscillations with the expected shape of a tree. In the second curve $\beta=0.02$. The magnetoCoulomb oscillations now are irregularly spaced. This effect becomes more pronounced when $\beta$ is increased further. For $\beta=0.08$ we obtain remarkable agreement with the measured data in Fig. 2(a) in Ref. 23. This calculated curve corresponds to $C_{\text {res }} \sim 10 \%$ of the total capacitance of the dot, which is a reasonable value. The agreement between experiment and calculation indicates the importance of the fielddependent capacitances between dot and reservoirs. The subject of mesoscopic, magnetocapacitances have also recently been discussed theoretically by Büttiker, Thomas, and Prêtre. $^{31}$

There is one important aspect of the data that remains unexplained. The two curves in Fig. 14 show that the data are hysteretic in the field direction. Note that the curves only differ in the field region $(5.1 \mathrm{~T}-5.7 \mathrm{~T})$. The two traces were completely reproducible and did not depend on the sweep rate of the field up to sweeps as slow as $30 \mathrm{~min}$ per T. We emphasize that data as in Fig. 14 are regularly observed. Also in dot I and II we observed a sharp change in the period of the magneto-Coulomb oscillations in this field region. The hysteresis, however, was sample dependent.

We think that the presence of disorder and localized states in the reservoirs is responsible for the hysteresis. Near a crossing in filling factor the self-consistent potential landscape in the reservoirs contains small compressible puddles 


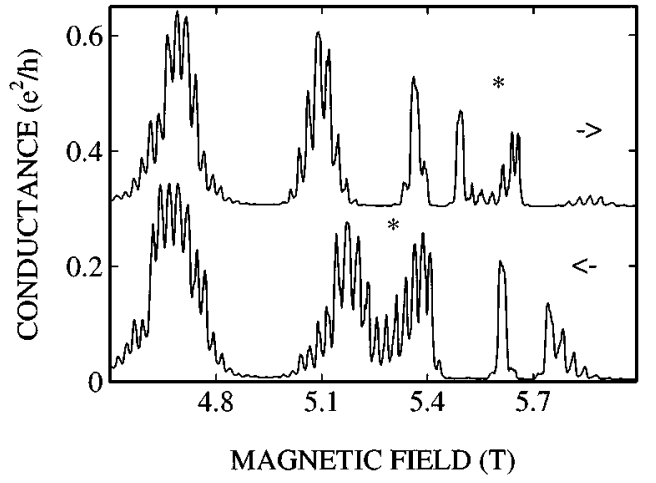

FIG. 14. Dependence of the magnetoconductance on sweep direction. The top curve has been measured while sweeping the field up and the bottom curve has been measured while sweeping down. Note that both curves show a dip in the magneto-Coulomb oscillations denoted by the asterisk. Measurements are performed on dot III.

of electrons (see also Ref. 32). Each puddle looks like our "dot-in-dot" picture of Fig. 1(a) where charging effects are important. Internal charging effects and the coupling between puddles may also be important. As we have discussed in this paper the typical time scale for (de)charging the puddles can be macroscopically long. Also, the exact puddle formation may be different on sweeping the field up compared to sweeping down. Both a long time scale and the exact puddle formation can be the origin for the observed hysteresis.

\section{CONCLUSIONS AND DISCUSSION}

The time-averaged data presented in this paper agree in detail with the earlier experiments by McEuen et al. ${ }^{6,7}$ Their self-consistent edge channel model provides a simple and elegant framework to explain our experiments. The new aspects of our experiment are in the data that has not been averaged over time. We have taken these data by using true dc measurement techniques instead of a commonly used near-zero-frequency lock-in technique.

The hysteresis that we observed in magnetoconductance sweeps seems to be related to the formation of small electron puddles in the reservoirs (Ref. 32). Since a dot is extremely sensitive for the charges in its environment, it is able to detect small differences in the exact puddle configuration between sweeping the magnetic field up or down. To resolve the internal structure of the reservoirs probably requires a scanning probe with which one can study Landau-level depopulation with spatial resolution.
In the switching experiments we used the dot as its own detector. Landau-level formation breaks the electron gas in the dot up in separate parts. These parts are so well separated that single-electron tunneling between them can have macroscopic time scales. We note that a time of $100 \mathrm{~s}$ has striking implications. If we assume that the drift velocity of the electrons along the circumference of the dot is about $10^{4} \mathrm{~m} / \mathrm{s}$ (Ref. 25) we obtain an anormous length scale of $1000 \mathrm{~km}$ before an electron is scattered to another quantum Hall state.

Our model, based on three energies $\left(E_{1}, E_{2}\right.$, and $\left.E_{12}\right)$ and some properties of a two-level system provides a quantitatively consistent explanation of the different experiments. We emphasize that this model is completely general and, in fact, also works for a dot treated as a black box. We have interpreted the parameters in terms of the self-consistent picture of two Landau levels in a dot. The parameters $E_{1}, E_{2}, E_{12}$, and $\delta$ have values that are reasonable for this picture. However, the time scales are so long that we think it cannot be simply explained from the width and height of the barrier formed by the incompressible strip. Other contributions to a long time scale may come from the dynamics of charge rearrangement. Many-body correlations such as in a Wigner molecule ${ }^{26}$ can strongly slow down such a rearrangement. Also, charge spreading in a magnetic field is expected to be very slow. ${ }^{33}$ In this respect, out long times may be related to the observed suppression of tunneling into quantum Hall states in a bulk 2DEG. ${ }^{34,35} \mathrm{We}$ also note the recent prediction $^{36}$ and observation ${ }^{37}$ of so-called Skyrmions in a bulk 2DEG. These works concluded that due to exchange interaction the two spin-resolved states of the lowest Landau level are more strongly mixed then previously expected. Although nothing is yet known about Skyrmions in confined structures this conclusion seems to conflict with our assumption of a well-separated core and ring structure. Therefore, more theoretical work along the lines of Refs. 38-40 is desired for either confirming our interpretation in terms of a separated ring and core, or for putting forward an alternative picture for a dot with internal charging energies.

\section{ACKNOWLEDGMENTS}

We thank C. Dekker, L. J. Geerligs, P. L. McEuen, C. T. Johnson, and J. E. Mooij for stimulating discussions, F. E. Cochius for assistance with the experiments, S. F. Godijn, W. Kool, and A. van der Enden for contributions to the device fabrication, and the Delft Institute for MicroElectronics and Submicron Technology for the use of their facilities. Financial support from FOM and the Royal Netherlands Academy of Arts and Sciences is gratefully acknowledged.
*Present address: Philips Research Laboratories, Prof. Holstlaan 4 (WY 5-01) 5656 AA Eindhoven, The Netherlands.

†Present address: Department of Physics, University of Nottingham, Nottingham NG72RD, United Kingdom.

${ }^{1}$ K. von Klitzing, G. Dorda, and M. Pepper, Phys. Rev. Lett. 45, 494 (1980).

${ }^{2}$ B. I. Halperin, Phys. Rev. B 25, 2185 (1982).

${ }^{3}$ M. Büttiker, Phys. Rev. B 38, 9375 (1988).
${ }^{4}$ B. J. van Wees, E. M. M. Willems, L. P. Kouwenhoven, C. J. P. M. Harmans, J. G. Williamson, C. T. Foxon, and J. J. Harris, Phys. Rev. B 39, 806 (1989); S. Komiyma, H. Hirai, S. Sasa, and S. Hiyamizu, ibid. 40, 12566 (1989); B. W. Alphenaar, P. L. McEuen, R. G. Wheeler, and R. N. Sacks, Phys. Rev. Lett. 64, 677 (1990).

${ }^{5}$ A. M. Chang, Solid State Commun. 74, 271 (1990); C. W. J. Beenakker, Phys. Rev. Lett. 64, 216 (1990). 
${ }^{6}$ P. L. McEuen, N. S. Wingreen, E. B. Foxman, J. Kinaret, U. Meirav, M. A. Kastner, Y. Meir, and S. J. Wind, Physica B 70, 70 (1993)

${ }^{7}$ P. L. McEuen, E. B. Foxman, J. M. Kinaret, U. Meirav, M. A. Kastner, N. S. Wingreen, and S. J. Wind, Phys. Rev. B 45, 11419 (1992).

${ }^{8}$ D. B. Chklovski, B. I. Shklovskii, and L. I. Glazman, Phys. Rev. B 46, 4026 (1992).

${ }^{9}$ I. K. Marmorkos and C. W. J. Beenakker, Phys. Rev. B 45, 11419 (1992).

${ }^{10}$ J. M. Kinaret and N. S. Wingreen, Phys. Rev. B 48, 11113 (1993).

${ }^{11}$ For a review, see Single Charge Tunneling, edited by H. Grabert and M. H. Devoret (Plenum, New York, 1991); for a recent review on quantum dots, see L. P. Kouwenhoven and P. L. McEuen, in Nano-Science and Technology, edited by G. Timp (AIP, New York, 1996).

${ }^{12}$ N. C. van der Vaart, M. P. de Ruyter van Steveninck, L. P. Kouwenhoven, A. T. Johnson, Y. Nazarov, C. J. P. M. Harmans, and C. T. Foxon, Phys. Rev. Lett. 73, 320 (1994).

${ }^{13}$ A. A. M. Staring, B. W. Alphenaar, H. van Houten, L. W. Molenkamp, O. J. A. Buyk, A. A. Mabesoone, and C. T. Foxon, Phys. Rev. B 46, 12869 (1992).

${ }^{14}$ T. A. Fulton, P. L. Gammel, and L. N. Dunkleberger, Phys. Rev. Lett. 67, 48 (1991).

${ }^{15}$ P. Lafarge, H. Pothier, E. R. Williams, D. Esteve, C. Urbina, and M. H. Devoret, Z. Phys. B 85, 327 (1991).

${ }^{16}$ For zero field data, see L. P. Kouwenhoven, N. C. van der Vaart, A. T. Johnson, W. Kool, C. J. P. M. Harmans, J. G. Williamson, A. A. M. Staring, and C. T. Foxon, Z Phys. B 85, 367 (1991).

${ }^{17}$ The dot conductance exceeds the conductance of the individual barriers. From additional measurements we found that upon increasing the temperature, the amplitude of the oscillations decreases. These are clear signatures for coherent resonant tunneling through discrete energy states; see Ref. 11.

${ }^{18} \mathrm{We}$ determined this conversion factor by measuring the Coulomb oscillations at nonzero source-drain voltage. We note that in Ref. 12 we estimated this conversion factor too low.

${ }^{19}$ N. C. van der Vaart, M. P. de Ruyter van Steveninck, L. P. Kouwenhoven, A. T. Johnson, Y. Nazarov, C. J. P. M. Harmans, and C. T. Foxon, in Quantum Dynamics of Submicron Structures, Vol. 291 of NATO Advanced Study Institute, Series E: Applied Science, edited by H. A. Cerdeira, B. Kramer, and G. Schön (Kluwer, Dordrecht, 1994).
${ }^{20}$ A. K. Evans, L. I. Glazman, and B. I. Shklovskii. Phys Rev. B 48, 11120 (1993).

${ }^{21}$ T. Heinzel, D. A. Wharam, J. P. Kotthaus, G. Bohm, W. Klein, G. Trankle, and G. Weimann, Phys. Rev. B 50, 15113 (1994).

${ }^{22}$ I. O. Kulik and R. I. Shekter, Zh. Eksp. Teor. Fiz, 68, 623 (1975) [Sov. Phys. JETP 41, 308 (1975); C. W. J. Beenakker, Phys. Rev. B 44, 1646 (1991).

${ }^{23}$ N. C. van der Vaart, M. P. de Ruyter van Steveninck, C. J. P. M. Harmans, and C. T. Foxon, Physica B 194-196, 1251 (1994).

${ }^{24}$ J. P. Bird, K. Ishibashi, M. Stopa, R. P. Taylor, Y. Aoyagi, and T. Sugano, Phys. Rev. B. 49, 11488 (1994).

${ }^{25}$ Similar magnetic depopulation has been seen in a dot with one or more extended edge channels. See B. J. van Wees et al., Phys. Rev. Lett 62, 2523 (1989); B. W. Alphenaar, A. A. M. Staring, H. van Houten, M. A. A. Mabesoone, O. J. A. Buyk, and C. T. Foxon, Phys. Rev. B 46, 76236 (1992).

${ }^{26}$ Y. V. Nazarov and A. V. Khaetskii, Phys. Rev. B 49, 5077 (1994).

${ }^{27}$ C. W. J. Beenakker, Phys. Rev. Lett. 64, 216 (1990).

${ }^{28}$ L. P. Kouwenhoven, B. J. van Wees, N. C. van der Vaart, C. J. P. M. Harmans, and C. T. Foxon, Phys. Rev. Lett. 64, 685 (1990); A. M. Chang and J. E. Cunningham, ibid. 69, 2114 (1992).

${ }^{29}$ S. Machlup, J. Appl. Phys. 25, 341 (1954).

${ }^{30}$ A similar discussion with a somewhat different interpretation has been given by M. Stopa, Y. Aoyagi, and T. Sugano, Phys. Rev. B 51, 5494 (1995).

${ }^{31}$ M. Büttiker, H. Thomas, and A. Prêtre, Phys. Lett. A 180, 364 (1993).

${ }^{32}$ D. B. Chklovskii, and P. A. Lee, Phys. Rev. B 48, 18060 (1993).

${ }^{33}$ I. L. Aleiner, H. U. Baranger, and L. I. Glazman, Phys. Rev. Lett. 74, 3435 (1995).

${ }^{34}$ R. C. Ashoori, J. A. Lebens, N. P. Bigelow, and R. H. Silsbee, Phys. Rev. Lett. 64, 681 (1990).

${ }^{35}$ J. P. Eisenstein, L. N. Pfeiffer, and K. W. West, Phys. Rev. Lett. 69, 3804 (1992).

${ }^{36}$ S. L. Sondhi, A. Karlhede, S. A. Kivelson, and E. H. Rezayi, Phys. Rev. B 47, 16419 (1993); H. A. Fertig, L. Brey, R. Cote, and A. H. MacDonald, ibid. 50, 11018 (1994).

${ }^{37}$ S. E. Barret, G. Dabbagh, L. N. Pfeiffer, K. W. West, and R. Tycko, Phys. Rev. Lett. 74, 5112 (1995).

${ }^{38}$ S. -R. E. Yang, A. H. MacDonald, and M. D. Johnson, Phys. Rev. Lett. 71, 3194 (1993).

${ }^{39}$ C. de C. Chamon and X. G. Wen, Phys. Rev. B 49, 8227 (1994).

${ }^{40}$ T. H. Stoof and G. E. W. Bauer, Phys. Rev. B 52, 12143 (1995). 